\title{
Study of Anderson Score Evaluation in the Diagnosis of Acute Appendicitis
}

\author{
Authors \\ Vijay Kumar Gupta ${ }^{1}$, Dr Sahjanand Prasad Singh ${ }^{2}$ \\ ${ }^{\mathbf{1}}$ MBBS, MS (Gen. Surg), Assistant Professor, Department of General Surgery, VIMS, Pawapuri, Nalanda, \\ Bihar-803115 \\ ${ }^{2}$ MBBS, MS(Gen. Surg), Associate Professor \& HOD, Department of General Surgery, VIMS, Pawapuri, \\ Nalanda, Bihar-803115 \\ *Corresponding Author \\ Dr Sahjanand Prasad Singh \\ Associate Professor \& HOD, Department of General Surgery, Vardhman Institute of Medical \\ Sciences(VIMS), Pawapuri, Nalanda, Bihar-803115 \\ Email: sahajsurgery.phulwarisharif@gmail.com
}

\begin{abstract}
Introduction: Acute appendicitis is one of the most common surgical emergency making up to 10\%of all emergency abdominal surgeries. It is difficult to diagnose acute appendicitis in young, elderly patients and female of reproductive age group because they have atypical presentation. No investigation is fool proof combination of clinical history, physical examination and laboratory studies lead to development of several scoring systems.

Material and Methods: 60 subjects of both age and sex presenting with pain in right iliac fossa and suspected to have acute appendicitis enrolled for the study.

Result: These findings were statistically significant. ( $p$ value <0.05) Sensitivity of the ANDERSON scoring system in the study was $76.6 \%$, specificity came out to be $100 \%$. The positive and negative values were $100 \%$ and $21.43 \%$ respectively. Apart from ALVARDO and modified ALVARDO score Anderson score (appendicitis inflammatory response score) was developed with better sensitivity and specificity for diagnosis of appendicitis

Conclusion: Anderson score (appendicitis inflammatory response score) was developed with better sensitivity and specificity for diagnosis of appendicitis.

Keywords: Acute Appendicitis, Anderson Score, Alvardo and modified ALVARDO score.
\end{abstract}

\section{Introduction}

Acute appendicitis is the most common surgical procedure done in operation theatre world wide. Overall risk of acute appendicitis in general population is $8.7 \%$ for males and $6.9 \%$ for females. Peak incidence occur between the ages of 10 to 30 years. Pain abdomen in right ileac fossa is the most common presenting symptom of acute appendicitis.
Associated symptoms include loss of appetite, nausea, vomiting and occasionally a low grade fever. Failure of early diagnosis can lead to increased risk of perforation and peritonitis, with increase in mortality and morbidity.

Diagnostic tool includes: Total leucocyte count (TLC), C - reactive protein (CRP), Ultrasonography (USG), plain skiagram of abdomen and Computed Tomography (CT) scan have been used in the 
diagnosis of acute appendicitis. Since no investigation is fool proof, combination of clinical history, physical examination and laboratory studies lead to development of several scoring systems to help the clinician in diagnosing acute appendicitis in the fastest and cheapest way. In 1986 Alvarado constructed a 10 point clinical scoring system, also known by the acronym MANTRELS for the diagnosis of Acute appendicitis based on sign and 4symptoms and certain diagnostic tests. In 1994 Kalan produced a modified Alvarado score [MAS]. Both the scoring systems have poor sensitivity and specificity when applied in middle eastern, elderly and women of reproductive age group. This has led to the development of ANDERSON SCORE (APPENDICITIS INFLAMMATORY RESPONSE SCORE).

Based on this scoring system patients are classified into three groups;

Group 1:- Score 0-4=low probability for acute appendicitis; these patients were discharged after initial assessment, with strict advice to come back to the same unit and hospital if symptoms persist or recur.

Group 2:- Score 5-8=intermediate probability for appendicitis; these patients require in hospital active observation with rescoring, imaging or diagnostic laproscopy. If the score dropped to less than 4, patients were discharged with advice to come back if symptoms recur or persists. Otherwise if score rose upto 9 or more they were operated

Group 3:- Score 9-12=high probability for appendicitis; urgent surgical exploration is recommended for this group.

Table-I: Various Parameters used in Anderson Score are as Follows

\begin{tabular}{|l|c|c|}
\hline VARIABLE & LEVEL & SCORE \\
\hline $\begin{array}{l}\text { Pain or tenderness in } \\
\text { right lower quadrant }\end{array}$ & & +1 \\
\hline Vomiting & & +1 \\
\hline Rebound tenderness or & Slight Moderate Strong & +1 \\
muscular defense & & +2 \\
& & +3 \\
\hline WBC Coun & $10-$ & $+1+2$ \\
\hline Proportion neutrophils & $70 \%-84 \% \geq 85 \%$ & $+1+2$ \\
\hline CRP concentration & $10-49 \mathrm{mg} / 1 \geq 50 \mathrm{mg} / 1$ & $+1+2$ \\
\hline Body temperature & $\geq 38.5 * \mathrm{C}$ & +1 \\
\hline
\end{tabular}

The patient is taken up for surgery once the diagnosis is established and emergency appendicectomy is performed. The confirmation of diagnosis was done by histopathology. The minimum criteria for acute appendicitis is the presence of neutrophils in mucosa, submucosa and laminapropria. Thus looking at distinct advantages in terms of precision of diagnosis, the current study is planned so as to reduce the rate of negative appendicectomy. It is reiterated that reduction of negative appendicectomy is of utmost importance in modern day surgical practice keeping in mind the reconstructive role of appendix in variety of situations and also to reduce the economic burden on patient

\section{Material and Methods}

After approval from ethical committee of VIMS, Pawapuri this study done in a total of 60 patients of both age and sex presenting with pain in right iliac fossa and suspected to have acute appendicitis based on typical history and clinical findings were enrolled for the study. Patients having appendicular lump were excluded

The patient of acute appendicitis admitted to the emergency and outdoor of surgery department were identified and informed consent was taken from the patient for inclusion in the study. Detailed history and examination was done. Special stress given on history of pain, migration of pain to right iliac fossa, nausea, vomiting, tenderness, rebound tenderness, guarding, fever. Rovsing's sign, Psoas's sign, Obturator'ssign etc was elicited and recorded. Based on history and examination, clinical diagnosis was made. After that, total leucocyte count, CRP estimation and Ultrasound was done. The cutoff value for white cell counts was taken as $11000 / \mathrm{mm}$. Anderson score was calculated depending upon clinical symptoms, signs and laboratory investigations. Emergency appendicectomy was done in patients of group III through Mcburney's, lanz incision and in difficult cases with Rutherford Morrison Grid iron incision under anaesthesia. Confirmation of acute appendicitis as the final diagnosis was obtained from histopathological 
examination of the resected appendix in the department of pathology. Patient who were managed conservatively and subsequently discharged were reviewed once in the surgery outpatient clinic a week after discharge.

\section{Observations and Result}

The data was analyzed using SPSS software version 17. The mean age of the patients in the study was 31.5 years. The mean age for females was 37.33 years and mean age for males was 29 years. Majority (70\%) of patients were less than 40 years, with peak incidence in the age group of 19-29 years. Out of 60 cases, there were $42(70 \%)$ males and 15 $(30 \%)$ females in the study with male to female ratio of $2.3: 1$.

Most of the patients (86\%) presented with symptoms within 48 hours whereas, $14 \%$ patients presented later than 48 hours.

The most common symptom was pain in right iliac fossa which was present in all the patients, nausea vomiting in $82 \%$ of patients. Anorexia was present in $90 \%$ of patients and fever was present in $40 \%$ patients.

Most common sign was tenderness at Mc Burney's point $(98 \%)$ followed by guarding $(82 \%)$. Rebound tenderness was present in considerable number of patients (54\%). Rovsing's sign was present in only $19 \%$.

Out of 60 patients in this study, 82\% patients had raised Total leukocyte count, CRP was raised in $94 \%$ patients.

Anderson's score diagnosed appendicitis correctly in $74 \%$ of patients in the study group. In most (87\%) of these patients, the white blood cell count was raised. In $28 \%$ of the patients with Anderson's score $<9$, the leukocyte count was normal. The finding was statistically significant ( $p$-value $<0.000067$ ).

Most patients (70\%) in the studied population belonged to younger age group and $71 \%$ of these patients were diagnosed as acute appendicitis by the Anderson's scoring system. This finding was statistically significant ( $\mathrm{p}$-value $<0.05$ ).

Out of the 60 patients with appendicitis 57 were operated and were diagnosed as acute appendicitis on histopathology. 3 patients were managed conservatively. The most commonly encountered type was acute diffuse suppurative appendicitis with periappendicitis followed by Exudative appendicitis with periappendicitis and Gangrenous appendicitis. There was an increase in ANDERSON SCORE with increase in histopathological severity. The mean score for acute appendicitis with periappendicitis, acute diffuse suppurative appendicitis with periappendicitis and acute gangrenous appendicitis were 6, 9.02 and 11 respectively.

According to ANDERSON SCORE, 46 patients were diagnosed to have appendicitis. Out of these 46 patients all have evidence of appendicitis histopathologically. No patient was falsely diagnosed to have appendicitis by ANDERSON scoring system.14 patients were diagnosed on AIRS to not having appendicitis, but 11 out of 14 were diagnosed as appendicitis which were missed by this scoring system.

These findings were statistically significant. (p value $<0.05)$ Sensitivity of the ANDERSON scoring system in the study was $76.6 \%$, specificity came out to be $100 \%$. The positive and negative values were $100 \%$ and $21.43 \%$ respectively.

\section{Discussion}

Acute appendicitis is one of the most common non traumatic abdominal surgical emergencies encountered in the world particularly in age group less than 30 years, but still remains diagnostic challenge despite availability of various diagnostic modalities. Acute appendicitis traditionally has been a clinical diagnosis and remains so to this day. The diagnosis may be difficult to make in many patients who may present with atypical signs and symptoms or an equivocal physical examination, particularly very young, elderly patients and females of reproductive age group. Delay in diagnosis can lead to increased morbidity and even mortality. To prevent delay in diagnosis various investigations have been tried but diagnosis of acute appendicitis is still clinical. Till date we have no laboratory parameters that could indicate or reliably point to 
presence or absence of acute appendicitis. The clinical diagnosis will remain the cornerstone in acute appendicitis; nevertheless, laboratory investigations provide significant complementary aid in diagnosis. Surgeon's good clinical assessment is considered to be the most important requisite in the diagnosis of appendicitis. Several other conditions can mimic this clinical condition. Only CECT can diagnose the condition with very high sensitivity and specificity but it is not feasible to have this investigation for each and every patient suspected to be having appendicitis, particularly in countries with limited resources like ours.

This has resulted in considerable research to identify clinical, laboratory, radiological parameters and scoring systems to guide the clinician to make a correct diagnosis, thereby reducing the delay in diagnosis and decreasing the rate of negative appendicectomies. There has been need of scoring system with acceptable sensitivity, specificity and negative appendectomy rate. One of the most commonly used is the Alvarado scoring system which incorporates symptoms, signs and 1314laboratory investigations to reach the diagnosis. Many studies in the literature are available regarding the diagnosis of acute appendicitis in western population but there is paucity of literature available regarding application of various diagnostic score for diagnosis of this very common disease in Indian population

The present study was aimed for "Evaluation of Anderson score in the diagnosis of acute appendicitis". This study was carried out at VIMS, Pawapuri during a period from July 2017 to November 2018. It included 50 patients presenting to accident and emergency department and Surgery Outpatient Department of VIMS, Pawapuri with complaint of pain right lower abdomen and who have been clinically diagnosed as acute appendicitis.

\section{Conclusions}

Anderson score (appendicitis inflammatory response score) is having better sensitivity and specificity for diagnosis of appendicitis.

\section{References}

1. Al-Hashemy AM, Seleem MI. Appraisal of the modified Alvarado score for acute appendicitis on adults. Saudi Med J 2004; 25 : 1229-31.

2. Ali N, Rasul S, Mehmood Z, Inamullah, Khan A. Value of total leucocyte count and Creactiveproteins in the diagnosis of acute appendicitis. J Surg Pak 2009;14:153-6.

3. Alvarado A. A practical score for the early diagnosis of acute appendicitis. Ann Emerg Med 1986; 15: 557-64.

4. Anderson RE. Meta-analysis of the clinical and laboratory diagnosis of appendicitis. $\mathrm{Br}$ J Surg. 2004;91:28-37.

5. Burger TO, Torbert HC. Problems in the diagnosis of acute appendicitis. Cal West Med 1939 Jan; 50(1):7-11.

6. Eriksson S, Granstrom L, Carlstrom A. The diagnostic value of repetitive preoperative analyses of C-reactive protein and total leucocyte count in patients with suspected acute appendicitis. Scand J Gastroenterol 1994;29:1145-9.

7. Horton MD, Counter SF, Florence MG, Hart MJ. A prospective trail of computed tomography and ultrasonography for diagnosing appendicitis in the atypical patient. Am J Surg 2000; 179:379-81.

8. Pal KM, Khan A. Appendicitis, a continuing challenge. J Pak Med Assoc. 1998; 48:18992.

9. Kanumba ES, Mabula JB, Rambaau P, Chalya PL. Modified Alvarado Scoring System as a diagnostic tool for Acute Appendicitis at Bugando Medical Centre, Mwanza, Tanzania. BMC Surg 2011; 11:4.

10. Rajendra B. Incidence of various physical signs in acute appendicitis. Indian J Surg 1998; 40:14.

11. Shafi SM, Afsheen M, Reshi FA. Total leucocyte count, C-reactive protein and neutrophil count: diagnostic aid in acute appendicitis. Saudi J Gasteroenterol 2009; 15:117-20. 
12. Samad A, Kumar B, Khanzada TW, Lohana D. Appropriate cut off value of Alvarado scores for patients undergoing appendectomy in Isra University Hospital, Hyderabad. Isra Med J 2009 Aug; 1(2):36-9.

13. S.M.M. de Castro, E. Ph. Stellar, B.A. van Wagensveld, B.C. Vrouenraets. Evaluaton of the Appendicitis Inflammatory Response Score for Patients with Acute Appendicitis. World J Surg 2012; 36(7): 1540-5.

14. WY, Ho YC, Chu KW, Yeung C. Leucocyte count and neutrophil percentage in appendicectomy for suspected appendicitis. Aust N Z J Surg 1989;59(5):395-8. 